\title{
A Microstrip-Line-Fed Suspended Square Slot Microstrip Antenna for Circular Polarization Operations
}

\author{
Onkar M. Mudegaonkar \\ PG Department \\ MBES's College of Engineering \\ Ambajogai, India, 431517
}

\author{
Veeresh G. Kasabegoudar \\ PG Department \\ MBES's College of Engineering \\ Ambajogai, India, 431517
}

\begin{abstract}
A microstrip line-fed suspended square slot antenna with parasitic patch is presented. The proposed antenna is excited by a microstrip line feed placed at the bottom side of the substrate. The antenna geometry gets excitation via EM coupling. In this structure circular polarization $(\mathrm{CP})$ is also obtained with suspended air gap and CP frequency can be switched between $4.5 \mathrm{GHz}$ to $5.5 \mathrm{GHz}$. Thus, a $3 \mathrm{~dB}$ axial ratio bandwidth of about $4.32 \%$ is obtained. This structure exhibitsimpedance bandwidth, which is over $20.83 \%$. The antenna was optimized with Ansoft's HFSS software and was validated through a prototype. Measured results fairly agree with the simulated data.
\end{abstract}

\section{Keywords}

Wide-slot antenna, circular polarization (CP)

\section{INTRODUCTION}

Planar antennas have become wide area of interest for the antenna designers because of their unique features like compact size, multiband operation etc. Number of researchers has reported their works on wideband and circularly polarized slot antennas [1-14]. Printed slot antennas are widely used in a variety of communication systems because wide-slot antennas have two orthogonal resonance modes [1]. Therefore, printed slot antennas have recently received a great deal of attention from researchers. Each slot shape requires a feed stub of appropriate shape. For example, in [2], a printed wide-slot antenna fed by a microstrip line with a fork-like tuning stub has been used to obtain broad bandwidth through the proper parameters of the fork-like tuning stub.

S. Wei et.al., [3] presented a square-patch-shape feed with round corners at four vertexes. In another work [4], a novel bandwidth enhancement technique for microstrip-line-fed wideslot antenna based on fractal techniques has been reported. Yet another work by W. Chen et. al. [5], two resonance modes and rotation angle (with respect to center of square wide slot) for enhancement of impedance bandwidth. Bandwidth enhancement can also be achieved by tapering the feeding Line [6].

The modifications include for circular polarization are adjusting the dimensions of the basic patch with one or more feeds, trimming the corners of a square patch, feeding the patch at adjacent sides, feeding the patch (rectangular) from its corner along the diagonal, and Cutting a slot inside the patch.Various slot combinations are reported to obtain compact/broadband circular polarization [7]. It is well known that transmitting and receiving antennas are less sensitive to their respective orientations when their radiation patterns are circularly polarized $(\mathrm{CP})$ [8]. The circularly polarized antennas are often utilized in radar, satellite, radio frequency identification (RFID), navigation, and sensor systems [9].

The main advantage of single-feed circularly polarized microstrip antennas is their simple structures that do not require an external polarizer [10]. They can therefore be realized more compactly by using less board space than the dual feed circularly polarized antennas. Many designs of single-feed circularly polarized microstrip antennas with square or circular patches have also been reported. To obtain compact circular polarization $(\mathrm{CP})$ operation, some designs by embedding a cross slot of unequal slot lengths in the circular patch or inserting slits of different lengths at the edges of a square patch have been proposed recently [10]. The well known method to obtain circular polarization using single-feed patch antenna is slotted, truncated corner of square patch antenna. Since the two neardegenerate orthogonal modes of equal amplitude and 90 phase difference were excited, the purity of polarization will be relatively less. For these single-feed patch antennas, the measured impedance bandwidth for a VSWR $\leq 2$ and $3 \mathrm{~dB}$ axial ratio bandwidth are less than $3 \%$ and $1 \%$, respectively [11].

The main advantage of circular polarization is its greater flexibility in orientation angle between transmitters and receivers against linear polarization, better mobility, weather penetration, reduction in multipath reflections, and other kinds of abilities of anti-interference. Hence, circularly polarized microstrip antennas find applications in radar, navigation, and satellite communication realms [12]. The configuration of the SWSA (Strip loaded wide slot antenna) with a circular slot and reduced ground plane, and it is fed by a microstrip line, where a substrate with a relative permittivity of 2.33 and thickness of $0.787 \mathrm{~mm}$ is used [13].

Antenna configuration has been explained in Section 2. Section 3 presents the optimization procedure. The experimental validation of the optimized geometry is presented in Section 4. Finally, the work is concluded in Section 5. 

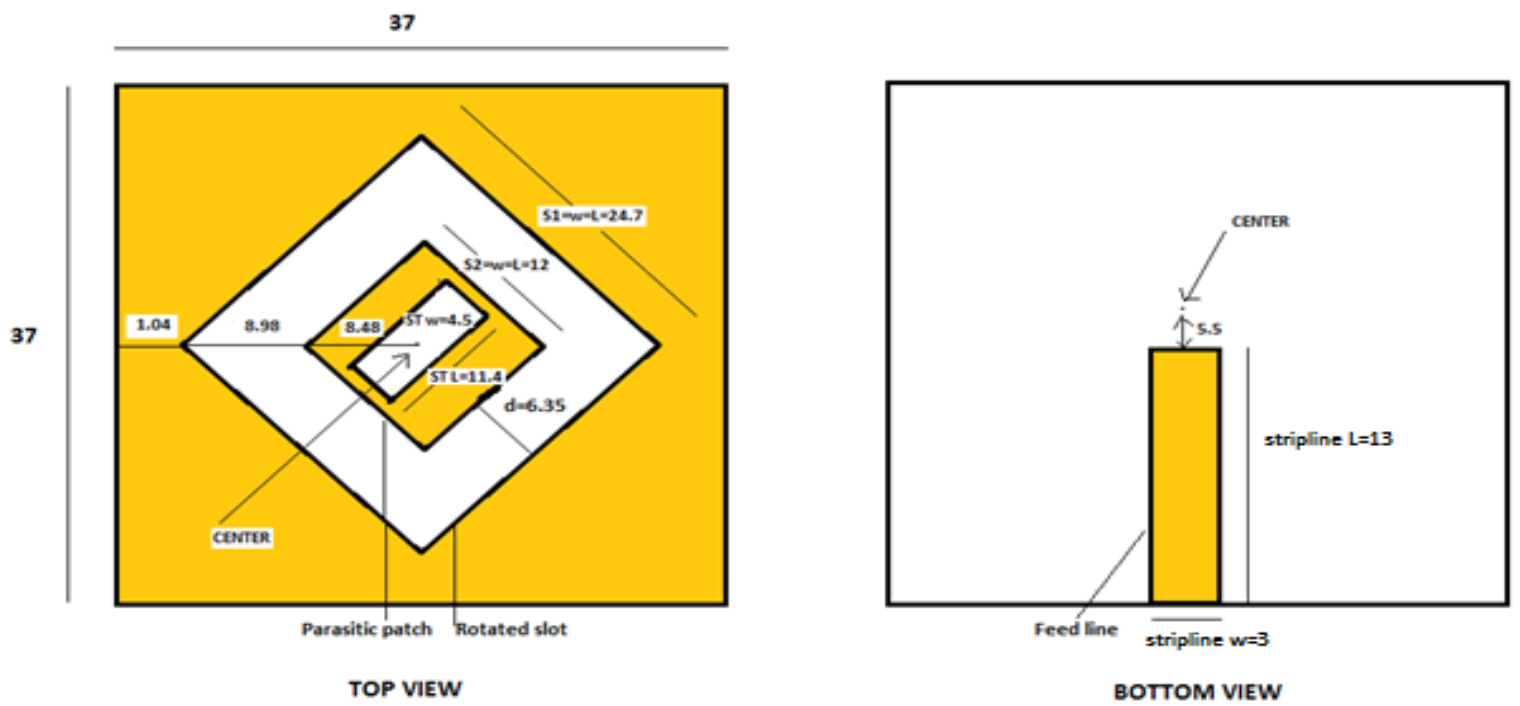

Fig1: Geometry and dimensions of antenna

\section{ANTENNA CONFIGURATION}

Fig 1 shows the geometry and dimensions of the proposed antenna, the printed wide slot is chosen to be a square in order to excite two modes with close resonant frequencies. For exciting the operating frequencies at around $4.5-5.5 \mathrm{GHz}$, the printed square slot rotated with an angle $45^{\circ}$ and is printed on an FR4 substrate of thickness $1.6 \mathrm{~mm}$ and $\varepsilon_{\mathrm{r}}=4.4$.

The ground plane is also chosen to be square with dimensions
$37 \mathrm{~mm} \times 37 \mathrm{~mm}$. This patch is fed by a $50 \Omega$ microstrip line with a simple tuning stub having a straight length of $L \mathrm{~mm}$, which is printed on the opposite side of the microwave substrate. For design simplicity, the width of the tuning stub is chosen to be the same as that of the $50 \mathrm{ohm}$ microstrip line. Simulated results show that square slot antenna with various dimension changes for circular polarization. The correct values can be optimized by observing the reflection coefficient $\&$ axial ratio of the antenna which is explained in detail in Section 3.

Table 1.Dimensions for the antenna geometry shown in Fig 1

\begin{tabular}{|l|c|c|c|c|c|c|c|c|c|c|c|}
\hline Parameters & $h$ & $S_{1}$ & $S_{2}$ & $L_{\text {off }}$ & $W(S R)$ & $L(S R)$ & $w$ & $L$ & $S T(L)$ & $S T(w)$ & $A G$ \\
\hline Value(mm) & 1.6 & 24.7 & 12 & 5.5 & 3 & 13 & 37 & 37 & 11.4 & 4.5 & 8 \\
\hline
\end{tabular}

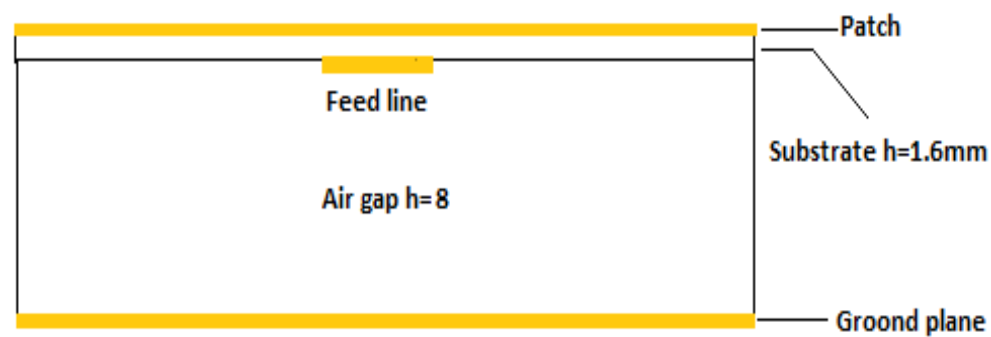

Fig 2: Layered geometry

\section{GEOMETRY OPTIMIZATION AND PARAMETRIC STUDY}

\subsection{Effect of Air Gap (AG)}

The proposed microstrip-line-fed square slot antenna has been constructed and studied on [1] HFSS, a software 3D tool by ANSOFT. By varying the parameter Air Gap (AG) in Fig 2, the measured return loss and axial ratio results of several design examples are shown in Fig 3. 


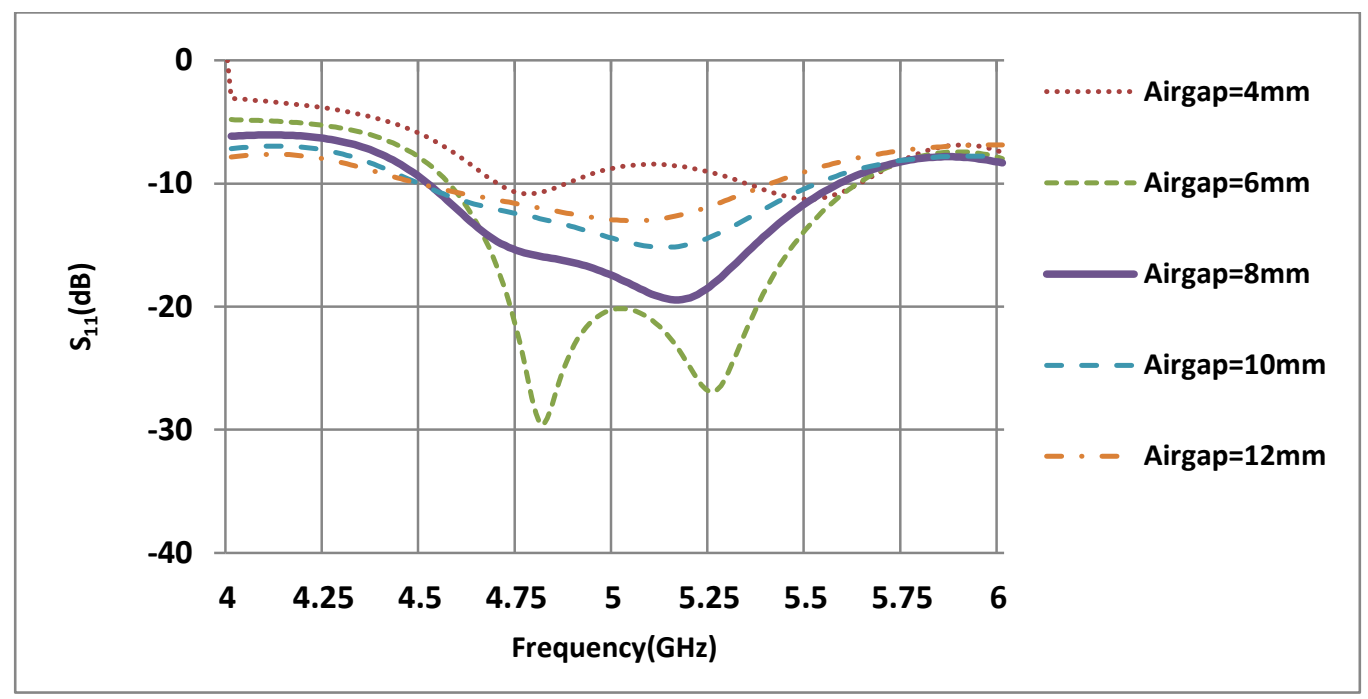

(a) Return loss vs. Frequency plot for variation in air gap

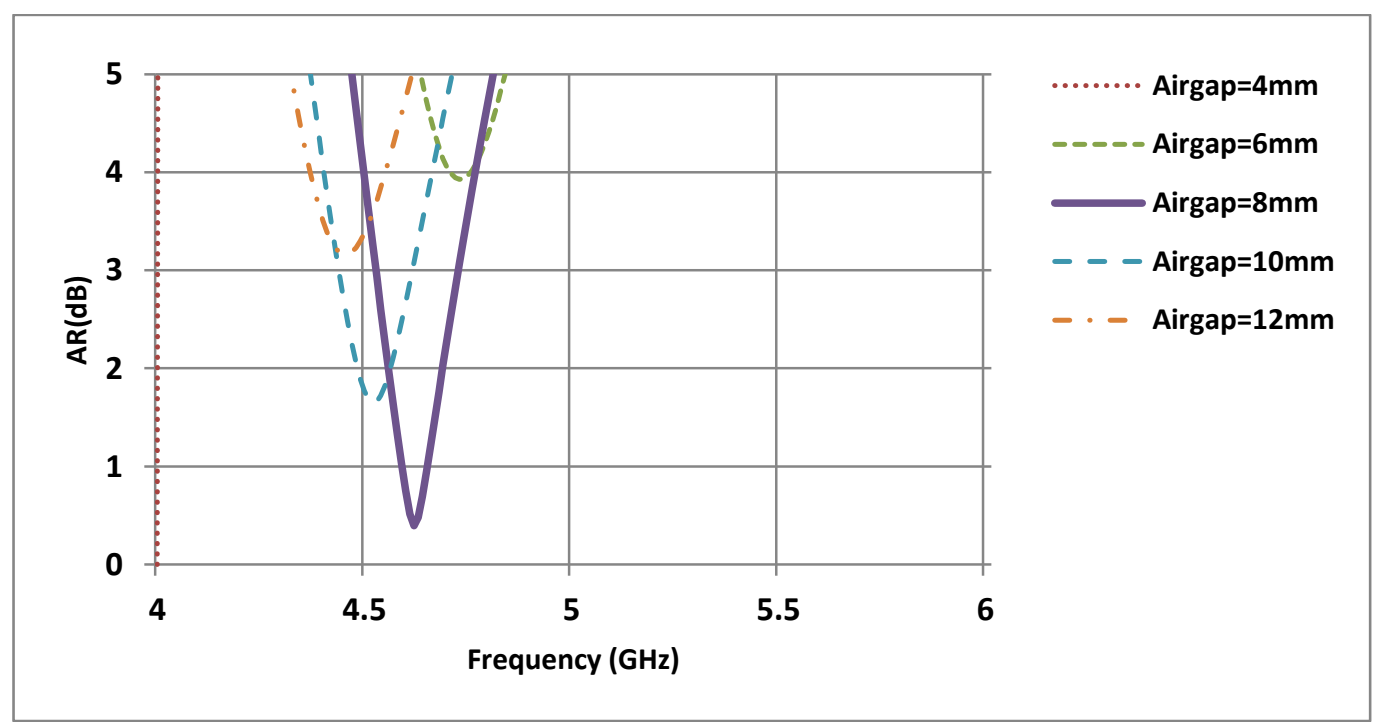

(b) Axial ratio vs. Frequency plot for variation in air gap

Fig 3: Return loss and axial ratio results with variation in air gap

Table 2.Effect of air gap (AG) on AR bandwidth

\begin{tabular}{|c|c|c|c|c|c|}
\hline Air Gap(mm) & 4 & 6 & 8 & 10 & 12 \\
\hline $\begin{array}{c}\text { Frequency Range } \\
\text { (AR< 3dB) (GHz) }\end{array}$ & -- & -- & $4.52-4.72$ & $4.43-4.61$ & -- \\
\hline AR BW (\%) & -- & -- & 4.32 & 3.98 & -- \\
\hline
\end{tabular}

This work uses the structure similar to the geometry proposed in [1]. However, besides enhancing the bandwidth we introduced circular polarization excitation which is required in most of the commercial wireless applications. It is observed that the reflection coefficient of antenna has a promising result at air gap height is $10 \mathrm{~mm}$. It is further improved if air gap dimensions are reduced till $8 \mathrm{~mm}$. It is also investigated that the decrease in the air gap height from $10 \mathrm{~mm}$ to $8 \mathrm{~mm}$ results in axial ratio improvement.

\subsection{Effect of Length and Width of Slot (ST)}

The proposed antenna with various parameters of length and width of slot (ST).The circular polarization (CP) can be obtained by cutting the slot inside the patch. The RHCP and LHCP pattern of the antenna is primarily depend on direction of that slot inside the patch. From Fig 4 (a) it is observed that the reflection coefficient is deeper long below $-10 \mathrm{~dB}$ in the given frequency range \& axial ratio bandwidth (ARBW) is also better than $11.1 \mathrm{~mm}$ length shown in Fig 4 (b). 


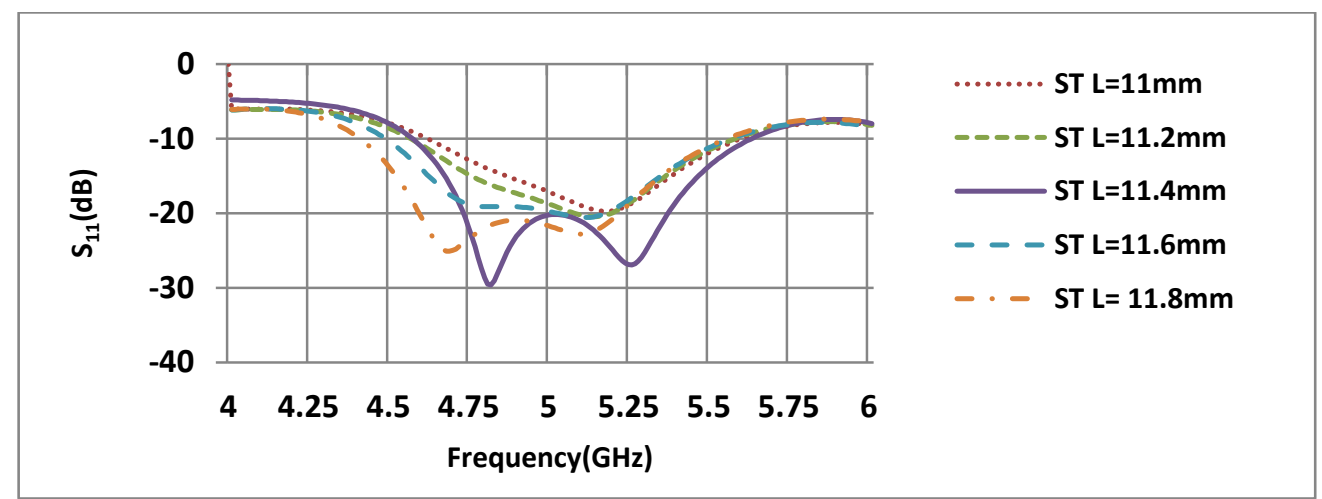

(a) Return loss Vs. Frequency plot for variation in slot (ST) length (L)

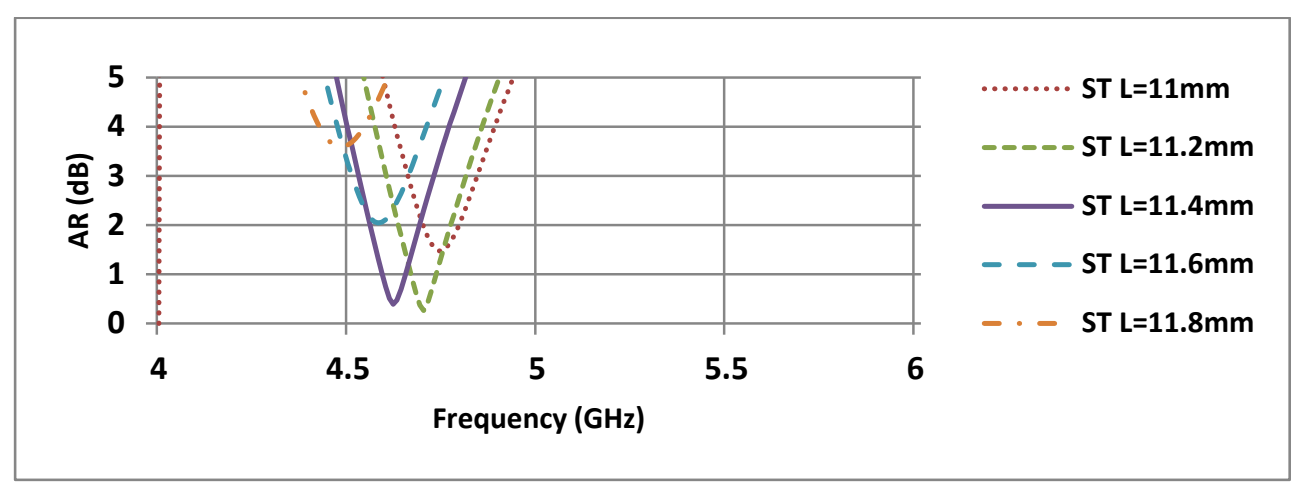

(b) Axial ratio Vs. Frequency plot for variation in slot (ST) length (L)

Fig 4: Return loss and axial ratio results with variation in slot length

The width of slot (ST) is varied from $1.5 \mathrm{~mm}$ to $7.5 \mathrm{~mm}$ in steps of $1.5 \mathrm{~mm}$. The return loss characteristics of this study are shown in Fig 5 (a). The maximum bandwidth is $20.83 \%$ for the slot width $\mathrm{w}=4.5 \mathrm{~mm}$ and other parameter kept constant. It is seen that the slot inside the patch is responsible for circular polarization the width of slot is also responsible for circular polarization. From Fig 5 (b) it is observed that as the slot width is increase from $1.5 \mathrm{~mm}$ to $4.5 \mathrm{~mm}$ the curve is falling down below $1 \mathrm{~dB}$ at the frequency $4.58 \mathrm{GHz}$. So the arrangement of slot width for the geometry is optimized for frequency $4.5 \mathrm{GHz}$. Table 3 and Table 4 show all summarized result.

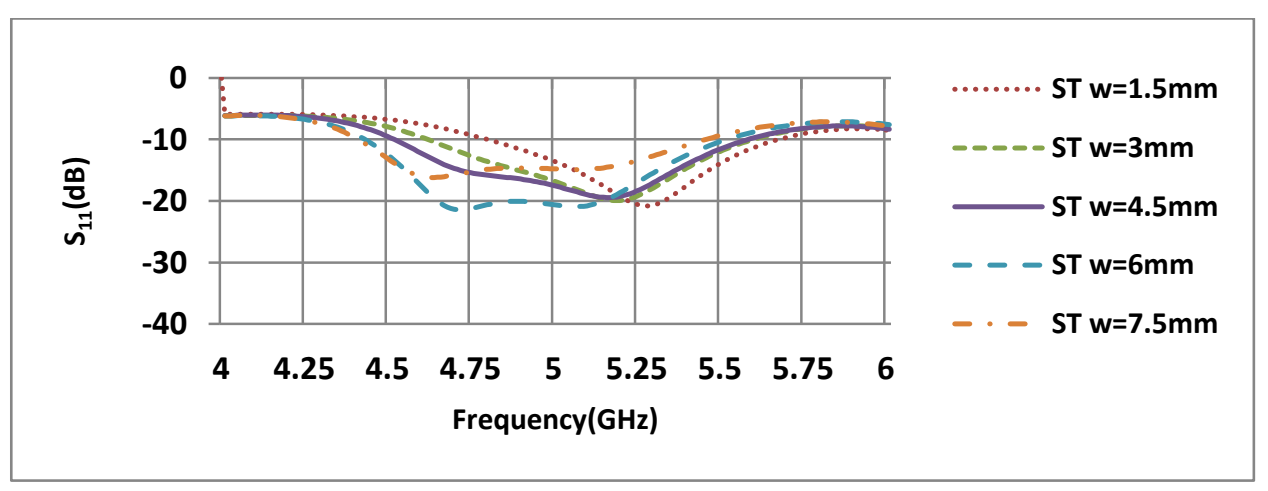

(a) Return loss Vs. Frequency plot for variation in slot (ST) width (w) 


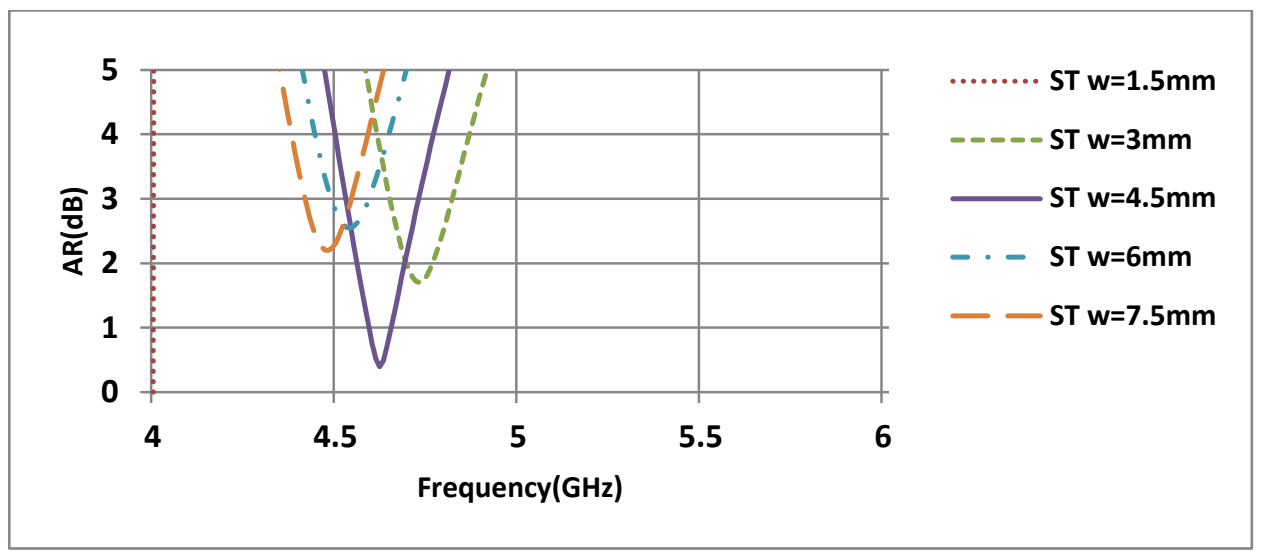

(b) Axial ratio Vs. Frequency plot for variation in slot (ST) width (w)

Fig 5: Return loss and axial ratio results with variation in slot width

Table 3.Effect of slot length on AR bandwidth

\begin{tabular}{|c|c|c|c|c|c|}
\hline Slot length (mm) & 11.0 & 11.2 & 11.4 & 11.6 & 11.8 \\
\hline $\begin{array}{c}\text { Frequency range } \\
\text { (AR< 3dB) (GHz) }\end{array}$ & $4.65-4.83$ & $4.59-4.78$ & $4.52-4.72$ & $4.43-4.61$ & -- \\
\hline AR BW (\%) & 3.79 & 4.05 & 4.32 & 3.98 & -- \\
\hline
\end{tabular}

Table 4. Effect of slot width on AR bandwidth

\begin{tabular}{|c|c|c|c|c|c|}
\hline Slot width (mm) & 1.5 & 3 & 4.5 & 6 & 7.5 \\
\hline $\begin{array}{l}\text { Frequency range } \\
(\text { AR< 3dB) (GHz) }\end{array}$ & -- & $4.64-4.81$ & $4.52-4.72$ & $4.48-4.58$ & $4.41-4.53$ \\
\hline AR BW (\%) & -- & 3.60 & 4.32 & 2.20 & 2.68 \\
\hline
\end{tabular}

\subsection{Effect of Strip Line (SR)}

A parasitic patch is fed by strip line at the bottom of the substrate. The strip line excites the patch located on other side of the substrate as shown in Fig 2. Strip line length is varied from $11 \mathrm{~mm}$ to $19 \mathrm{~mm}$ for better AR bandwidth. It is observed from Fig 6 that the geometry gives the optimized result at length $=13 \mathrm{~mm}$ by other parameters kept constant. Return loss characteristics and axial ratio show optimized bandwidth characteristics for $\mathrm{L}=13 \mathrm{~mm}$. All these results are summarized in Table 5.

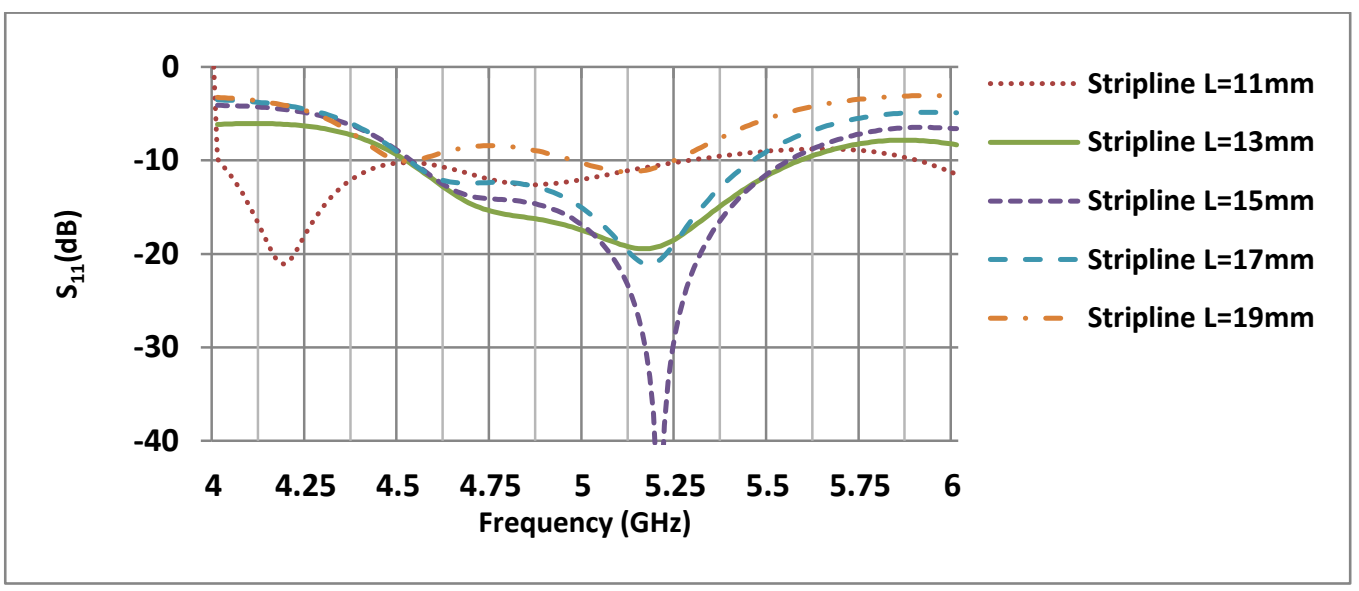

(a) Return loss Vs. Frequency plot for variation in stripline (SR) length 


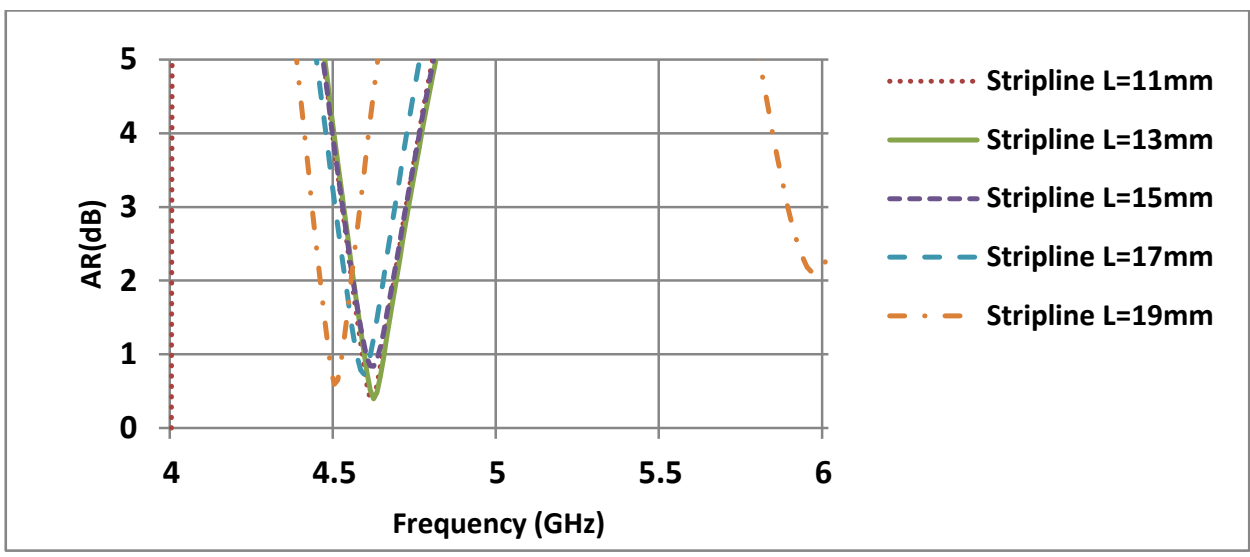

(b) Axial ratio Vs. Frequency plot for variation in strip (SR) length

Fig 6: Return loss and axial ratio results with variation in strip length

Table 5: Effect of strip length on AR bandwidth

\begin{tabular}{|c|c|c|c|c|c|}
\hline Stripline (SR) (mm) & 11 & 13 & 15 & 17 & 19 \\
\hline $\begin{array}{l}\text { Frequency range } \\
(\mathrm{AR}<3 \mathrm{~dB})(\mathbf{G H z})\end{array}$ & $4.52-4.71$ & $4.52-4.72$ & $4.52-4.71$ & $4.49-4.67$ & $4.42-4.57$ \\
\hline AR BW (\%) & 4.12 & 4.32 & 4.12 & 3.93 & 3.34 \\
\hline
\end{tabular}

\section{EXPERIMENTAL VALIDATION OF THE GEOMETRY AND DISCUSSIONS}

The geometry shown in Fig 7 with its optimizeddimensions presented in Table 1 was fabricated and tested.The substrate used for the fabrication is the FR4 glassepoxy with dielectric constant of 4.4 and height equal to $1.6 \mathrm{~mm}$. A photograph of thefabricated prototype is shown in Fig 7. Return losscomparisons of measured and simulated values aredepicted in Fig 8. From Fig 8 it may be noticed that the measured results fairly agreewith the simulated return loss characteristics. Radiation patterns across the CP band are shown in Fig 9, and from these patterns it may be noticed that LHCP \& RHCP patterns exhibit similar radiation characteristics.

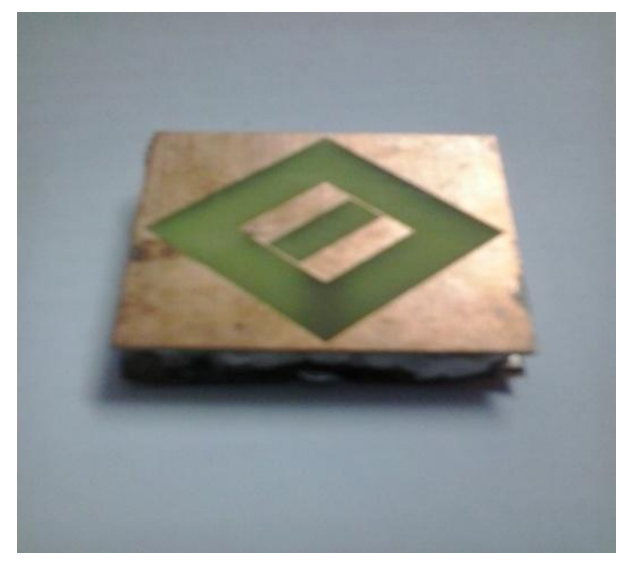

Fig 7: Photograph of the fabricated antenna

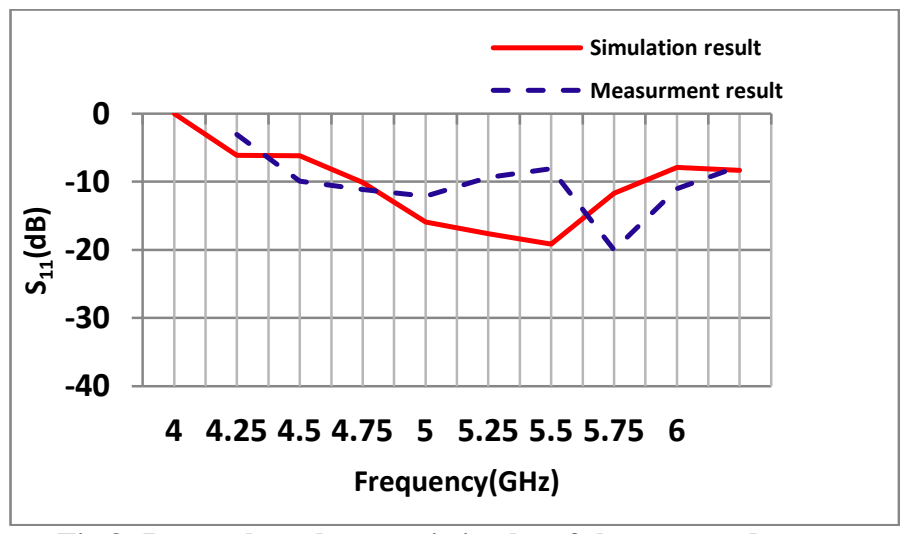

Fig 8: Return loss characteristic plot of the proposed antenna shown in Fig 1

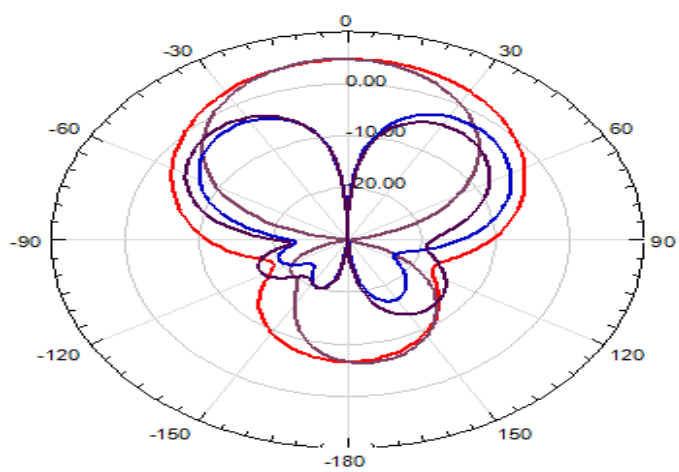

(a) $4.5 \mathrm{GHz}$ 


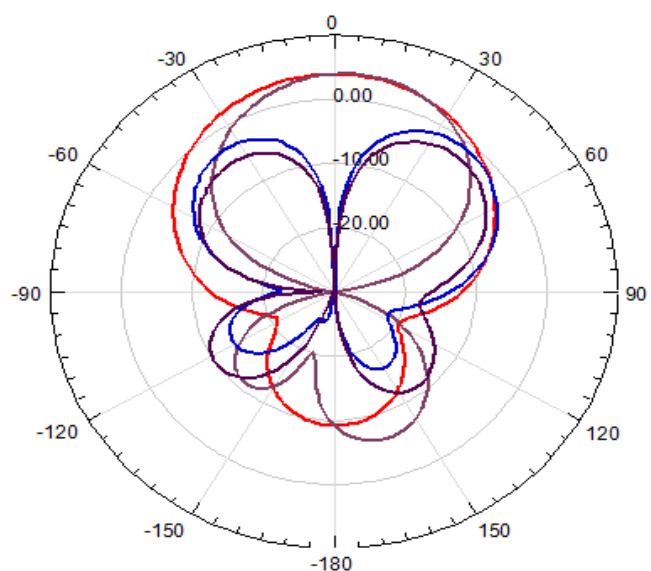

(b) $5 \mathrm{GHz}$

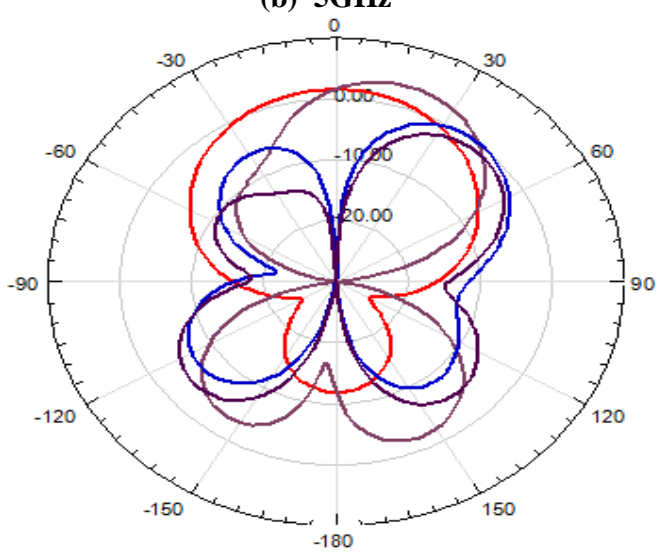

(c) $5.5 \mathrm{GHz}$

Fig 9: Radiation pattern at different frequencies in AR operating band

\section{CONCLUSION}

In this paper, the microstrip line fed printed wide slot microstrip antenna was presented for circular polarization. A diagonal slot was used to excite circular polarization and slot dimensions were optimized to maximize the AR bandwidth. The proposed geometry exhibits the return loss less than $-10 \mathrm{~dB}$ and the impedance bandwidth is $21 \%$. The geometry has also AR bandwidth of $4.32 \%$. By changing the height of air gap and slot dimensions LHCP and RHCP operation may be obtained by keeping other parameters constant. Also, further scope of the work includes further enhancement of $\mathrm{CP}$ and impedance bandwidths. Furthermore, the antenna has to be analytically modeled to understand its behavior for geometrical changes.

\section{REFERENCES}

[1] Y.Sung, "Bandwidth enhancement of a microstrip line-fed printed wide-slot antenna with a parasitic center patch," IEEE Trans. on Antennas and Prop., vol. 60, no. 4, pp. 1712-1716, April 2012.

[2] Jia-Yi Sze and Kin-Lu Wong, "Bandwidth enhancement of a microstrip-line-fed printed wide-slot antenna," IEEE Trans. on Antennas and Prop., vol. 49, no. 7, pp. 98-112,
July 2001.

[3] Shi-Wei Qu, ChengliRuan and Bing-Zhong Wang, "Bandwidth enhancement of wide-slot antenna fed by CPW and microstrip line," IEEE Antennas and Wireless Prop. Letters, vol. 5, pp. 15-17, 2006.

[4] Wen-Ling Chen, Guang-Ming Wang, and Chen-Xin Zhang, "Bandwidth enhancement of a microstrip-line-fed printed wide-slot antenna with a fractal-shaped slot," IEEE Trans. on Antennas and Prop., vol. 57, no. 7, pp. 21762179, July 2009.

[5] Jen-Yea Jan and Jia-Wei Su, "Bandwidth enhancement of a printed wide-slot antenna with a rotated slot," IEEE Trans. on Antennas and Prop., vol. 53, no. 6, pp. 21112114 June 2005.

[6] Pengcheng Li, Jianxin Liang and Xiaodong Chen, "Study of printed elliptical/circular slot antennas for ultrawideband applications," IEEE Trans. on Antennas and Prop., vol. 54, no. 6, pp. 1670-1675, June 2006.

[7] XiulongBao and M. J. Ammann, "Dual-frequency dualsense circularly-polarized slot antenna fed by microstrip line,', IEEE Trans. on Antennas and Prop., vol. 56, no. 3, pp. 645-649, March 2008.

[8] Ka Ming Mak and Kwai Man Luk, "A circularly polarized antenna with wide axial ratio beamwidth,"IEEE Trans. on Antennas and Prop., vol. 57, no. 10, pp. 3309-3312, October 2009.

[9] Christina F. Jou, Jin-Wei Wu, and Chien-Jen Wang, "Novel broadband monopole antennas with dual-band circular polarization," IEEE Trans. on Antennas and Prop., vol. 57, no. 4, pp. 1027-1034, April 2009.

[10] Jui-Han Lu, Chia-Luan Tang and Kin-Lu Wong, "Singlefeed slotted equilateral-triangular microstrip antenna for circular polarization," IEEE Trans. on Antennas and Prop., vol. 47, no. 7, pp. 1174-1178, July 1999.

[11] Yi-Fang Lin, Hua-Ming Chen and Shih-Chieh Lin, “A new coupling mechanism for circularly polarized annular-ring patch antenna," IEEE Trans. on Antennas and Prop., vol. 56, no. 1, pp. 11-16, January 2008.

[12] Yunxue Xu, Shuxi Gong, and Tao Hong, "Circularly polarized slot microstrip antenna for harmonic suppression," IEEE Antennas and Wireless Prop. Letters, vol. 12, pp. 472- 475, 2013.

[13] Shi-Wei Qu, Jia-Lin Li, Jian-Xin Chen, and QuanXue, "Ultrawideband strip-loaded circular slot antenna with improved radiation patterns," IEEE Trans. on Antennas and Prop., vol. 55, no. 11, pp. 3348-3353, November 2007.

[14] W.K.Lee, S.M.Kim, and W.G.Yang, "Single-feed multiple slotted triangular microstrip antenna for dual-bandcircular polarization"Proceedings of ISAP 2005, Seoul, Korea. 